\title{
PG 1325+101 and PG 2303+019: Two new large amplitude subdwarf B pulsators ${ }^{\star}$
}

\author{
R. Silvotti ${ }^{1}$, R. Østensen ${ }^{2,3}$, U. Heber ${ }^{4}$, J.-E. Solheim ${ }^{3}$, S. Dreizler ${ }^{5}$, and M. Altmann ${ }^{4,6}$ \\ 1 Osservatorio Astronomico di Capodimonte, via Moiariello 16, 80131 Napoli, Italy \\ 2 Isaac Newton Group of Telescopes, 37800 Santa Cruz de La Palma, Canary Islands, Spain \\ e-mail: roy@ing.iac.es \\ 3 Institutt for Fysikk, Universitetet i Troms $\varnothing$, 9037 Troms $\varnothing$, Norway \\ e-mail: jan-erik.solheim@phys.uit.no \\ 4 Dr. Remeis-Sternwarte, Astronomisches Institut der Universität Erlangen-Nürnberg, 96049 Bamberg, Germany \\ e-mail: ai03@sternwarte.uni-erlangen.de \\ 5 Institut für Astronomie und Astrophysik, Sand 1, 72076 Tübingen, Germany \\ e-mail: dreizler@astro.uni-tuebingen.de \\ 6 Sternwarte der Universität Bonn, Auf dem Hügel 71, 53121 Bonn, Germany \\ e-mail: maltmann@astro.uni-bonn.de
}

Received 6 November 2001 / Accepted 3 December 2001

\begin{abstract}
We report the detection of short period oscillations in the sdB stars PG $1325+101(B=13.8)$ and PG 2303+019 (alias HS 2303+0152, B = 16.0) from time-series photometry made at the Nordic Optical Telescope of a sample of 21 candidates. Both stars are multi-mode pulsators with at least three distinct periods in the range $100-140 \mathrm{~s}$, and relatively large amplitudes up to 2.6 and $1.6 \%$ respectively. Moreover PG $1325+101$ shows the shortest pulsation period ever registered among sdBV stars, $68.9 \mathrm{~s}$, which corresponds to the first harmonic of the main signal. Following previous temperature and gravity determinations for PG $1325+101\left(T_{\text {eff }}=34500 \mathrm{~K}\right.$, $\log g=6.1)$ and our NLTE model atmosphere analysis for PG $2303+019\left(T_{\text {eff }}=35200 \mathrm{~K}, \log g=5.7\right)$, both stars are well inside the theoretical sdBV instability strip.
\end{abstract}

Key words. stars: subdwarfs - stars: oscillations - stars: individual: PG 1325+101, PG 2303+019

\section{Introduction}

The discovery of the first subdwarf B (sdB) pulsator by Kilkenny et al. (1997) has opened a new attractive possibility of probing the internal structure of these stars using seismological methods. At about the same time of the discovery of the first pulsator, the existence of this new "sdBV" (=sdB Variable) instability strip, at effective temperatures below $\sim 30000 \mathrm{~K}$, was suggested by

Send offprint requests to: $\mathrm{R}$. Silvotti,

e-mail: silvotti@na.astro.it

* Based on observations obtained at the Nordic Optical Telescope, operated on the island of La Palma jointly by Denmark, Finland, Iceland, Norway, and Sweden, in the Spanish Observatorio del Roque de los Muchachos of the Instituto de Astrofisica de Canarias; and at the GermanSpanish Astronomical Center, Calar Alto, operated by the Max-Plank-Institute für Astronomie Heidelberg jointly with the Spanish National Commission for Astronomy.
Charpinet et al. (1996), who showed that an opacity bump associated with iron ionization was able to drive pulsations in these stars. More recent models indicate that the instability strip is centered around $33000 \mathrm{~K}$ (Charpinet et al. 1997), in good agreement with the observations. In the last three years, the number of discoveries of new sdB pulsators has rapidly increased; presently data on $25 \mathrm{sdBV}$ stars have been published (for reviews see O'Donoghue et al. 1999; Charpinet et al. 2001; additional discoveries are from Piccioni et al. 2000; Østensen et al. 2001a,b; Dreizler et al. 2001). The observational characteristics of the sdBV stars are rather similar to those of the pulsating white dwarfs: short pulsation periods (between $\sim 1$ and $10 \mathrm{~min}$ ), multimode behaviour, and small amplitudes (typically a few $\%$ or less).

The two new sdB pulsators reported in this paper, PG $1325+101(B=13.8)$ and PG $2303+019(B=16.0)$, are the latest discoveries of our search programme at the Nordic Optical Telescope (NOT), which has already 


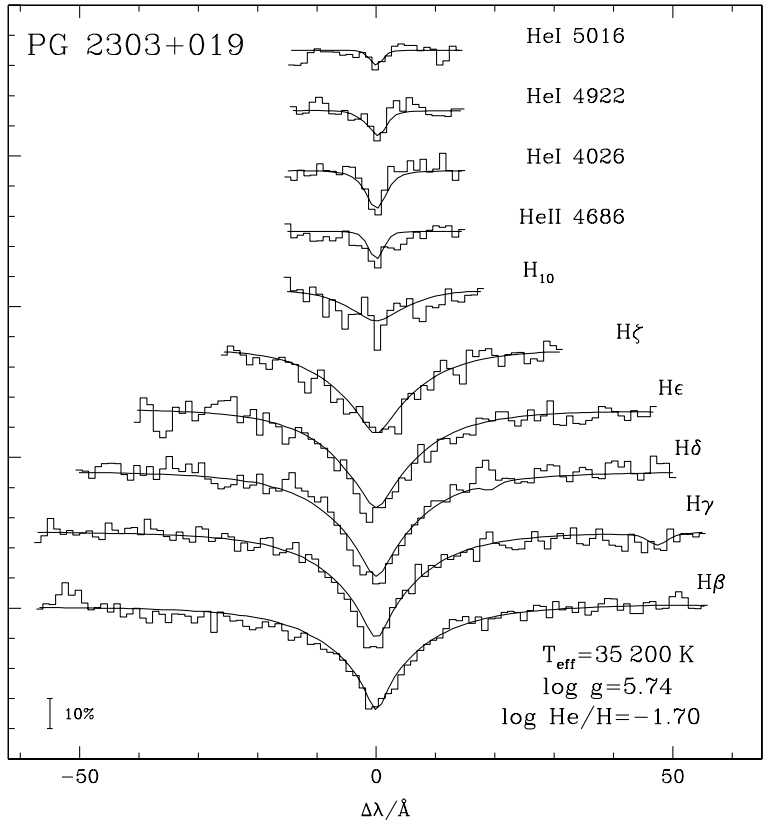

Fig. 1. Model fits to the Balmer and He line profiles in our time-averaged optical spectrum of PG 2303+019 using NLTE model atmospheres (see the text for more details).

produced 10 new sdB pulsators (Silvotti et al. 2000; Østensen et al. 2001a,b and this work) from a set of about 120 candidates investigated for photometric stability. These targets have been selected based on their spectroscopic temperatures from the Hamburg Schmidt (Hagen et al. 1995) and Palomar Green (Green et al. 1986) surveys. PG 2303+019, which was classified as an sdB star in the Palomar Green catalog, was recently rediscovered as an sdOB star (HS 2303+0152) during our followup spectroscopy of faint blue stars from the Hamburg Schmidt survey. The following astrometric and photometric measurements were obtained on Sep. 12, 1999 at the Calar Alto $1.2 \mathrm{~m}$ telescope: $\alpha(2000)=23^{\mathrm{h}} 06^{\mathrm{m}} 22.4^{\mathrm{s}}$, $\delta(2000)=+02^{\mathrm{d}} 09^{\mathrm{p}} 06^{\mathrm{s}} ; V=16.23, B=15.96, R=16.31$ (with errors of $\sim 0.02 \mathrm{mag}$ ).

\section{Spectroscopic characteristics}

The atmospheric parameters of PG $1325+101$ are known from the literature: Saffer et al. (1994) obtained $T_{\text {eff }}=$ $34500 \pm 1000 \mathrm{~K}, \log g=6.11 \pm 0.15, N(\mathrm{He}) / N(\mathrm{H})=$ $0.021 \pm 0.010$ from least-squares fitting of Balmer lines with LTE models. Slightly different results were obtained by O'Donoghue et al. (1998) with a similar technique: $T_{\text {eff }}=$ $35900 \pm 250 \mathrm{~K}, \log g=5.90 \pm 0.05$ (or $T_{\text {eff }}=34800 \pm 300$, $\log g=5.82 \pm 0.07$ using a different grating). Moreover PG 1325+101 does not show any significant radial velocity variation (Saffer et al. 1998).

For PG 2303+019 we obtained a medium resolution $(2.4 \AA)$ spectrum at the Calar Alto $3.5 \mathrm{~m}$ telescope with the TWIN spectrograph (grating T04, blue channel, $\lambda_{\mathrm{c}}=$ $4535 \AA$ ) on Sep. 15, 1999, using an exposure time of
1800 s. Besides the H Balmer lines, HeI lines and He II $4686 \AA$ were also detected. A grid of synthetic spectra derived from $\mathrm{H}-\mathrm{He}$ line blanketed NLTE model atmospheres (Napiwotzki 1997) was matched to the data to simultaneously determine effective temperature, surface gravity and He abundance (Heber et al. 2000). No set of parameters could be obtained that is consistent with both the Balmer line profiles and the helium ionisation equilibrium. In order to match the He ionisation equilibrium, the effective temperature needs to be higher by more than $2000 \mathrm{~K}$ than that one derived from the Balmer lines. A similar result was obtained for PG $1219+534$ from high resolution spectroscopy (Heber et al. 2000). The latter is almost a spectroscopic twin to PG 2303+019, having almost the same effective temperature, almost the same (relatively high) He abundance and a slightly larger gravity. Despite of a detailed investigation, Heber et al. (2000) could not find a solution to the discrepant results in the case of PG 1219+534 and adopted the average parameters derived from He ionisation equilibrium and Balmer line profiles respectively. Doing the same for PG 2303+019, we obtain $T_{\text {eff }}=35200 \pm 1500 \mathrm{~K}, \log g=5.74 \pm 0.15$ and $\log (\mathrm{He} / \mathrm{H})=-1.7 \pm 0.1$. The compromise fit is displayed in Fig. 1.

\section{Time-series photometry}

The time-series observations were obtained at the NOT with the Andalucia Faint Object Spectrograph and Camera $\left(\mathrm{ALFOSC}^{1}\right)$, modified with our own control software to be able to observe in high-speed multi-windowing mode. For each target, three or more reference stars were used. For PG $1325+101$ a Bessell B-band filter (NOT $\# 8^{1}$ ) was used, whereas for PG $2303+019$, due to its faintness, we used a wider filter (from $B$ to $R$ ), referred to as the $W$ filter $\left(\mathrm{NOT} \# 92^{1}\right)$. Exposure and cycle times are listed in Table 1, together with the log of the observations.

The data were reduced on-line using the Real Time Photometry (RTP) program developped by one of us (Østensen 2000). After the observations, a more accurate reduction was done optimizing the different steps. The processing of the data includes bias and flat-field

Table 1. Time-series photometry: log of the observations.

\begin{tabular}{lccccc}
\hline Object & $\begin{array}{c}\text { Date } \\
2001\end{array}$ & $\begin{array}{c}T_{\exp } \\
(\mathrm{s})\end{array}$ & $\begin{array}{c}T_{\text {cycle }} \\
(\mathrm{s})\end{array}$ & $\begin{array}{c}\text { Start } \\
(\mathrm{UT})\end{array}$ & $\begin{array}{c}\text { Length } \\
(\mathrm{s})\end{array}$ \\
\hline PG 1325+101 & Jul. 27 & 13.2 & 16.0 & $22: 12: 46$ & 5568 \\
& Jul. 28 & 10.3 & 14.0 & $21: 18: 59$ & 8372 \\
PG 2303+019 & Jul. 26 & 25.4 & 30.0 & $03: 10: 05$ & 9100 \\
& Jul. 27 & 25.6 & 30.1 & $02: 44: 37$ & 3240 \\
& Jul. 28 & 20.4 & 25.0 & $03: 29: 13$ & 5560 \\
& Jul. 29 & 20.4 & 25.0 & $03: 07: 39$ & 5260 \\
\hline
\end{tabular}

1 You can look at http://www.not.iac.es for more details. 

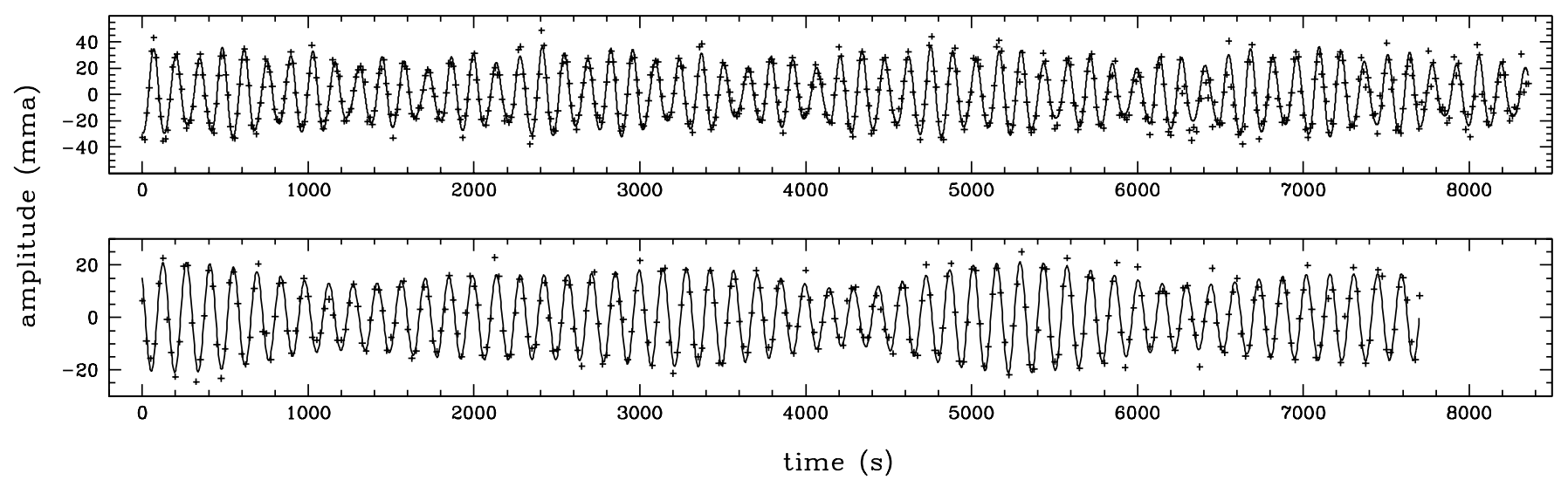

Fig. 2. Light curves of PG 1325+101 (top) and PG 2303+019 (bottom). For each star the longest run obtained in the last night (July 28-29) is shown. The continuous lines represent the multi-sinusoidal functions obtained from the least-square fits of all the available nights (see Table 2).

correction, sky subtraction, extinction correction and aperture photometry using optimal apertures that track each star geometrical center. Differential photometry is then obtained by means of the best combination of the available reference stars, using different apertures; at the end of this process, the best apertures are selected with a criterium that maximizes the $S / N$ ratio of the highest peak in the Fourier Transform (FT). The best results are obtained with apertures ranging between 3.4 and 4.2 arcsec. More details about observational techniques and data

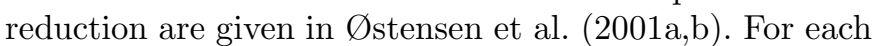
target, the light curve of the longest run is shown in Fig. 2.

The amplitude spectra of the two stars on each individual night are shown in Fig. 3. The temporal analysis of the data was performed using a prewhitening technique, i.e.: subtract the main signal from the data, calculate the FT of the residuals, extract the secondary frequency and repeat the procedure until the residuals were close to the noise level. In Fig. 4 the amplitude spectra of the two stars are shown at each step of the prewhitening procedure. At the end, a non-linear least-square sinusoidal fit was applied in order to optimize frequencies and amplitudes. This technique was repeated on each data set and also on the larger sets obtained when combining all the available nights. The results are listed in Table 2 .

\section{Discussion}

The two new sdB pulsators presented in this paper are located well inside the region of the $\left(T_{\text {eff }}, \log g\right)$ plane where the sdB pulsators are expected. Both stars show several pulsation modes, with quite stable amplitudes: from Table 2, the night to night amplitude variations of the main signals (which are less sensitive to the noise than the low amplitude peaks) are less than $4 \%$ for PG $2303+019$ and almost zero for PG $1325+101$, for which, however, data from only two nights are available. From the lower panels of Fig. 4 one can see that residual power is present for both stars, at about $6.3 \mathrm{mHz}$ for $\mathrm{PG} 1325+101$ and
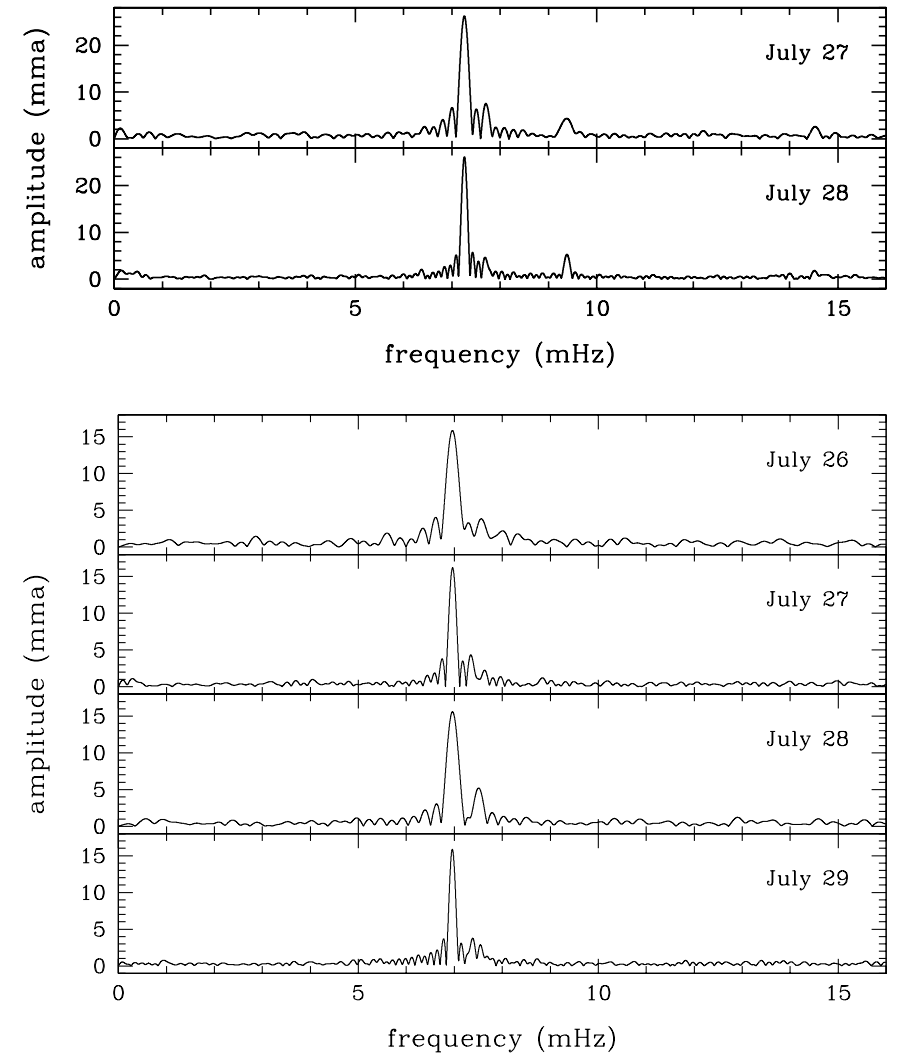

Fig. 3. Temporal spectra of PG $1325+101$ (top) and PG 2303+019 (bottom) in each individual night of observation.

near $8.8 \mathrm{mHz}$ for PG 2303+019, suggesting that further low amplitude frequencies might be present. Hence both stars might become good candidates for seismological studies as a large number of frequencies is crucial for the identification of the pulsation modes. Longer observing runs will be required in order to check whether further low amplitude signals are actually present and to resolve possible multiplets. 

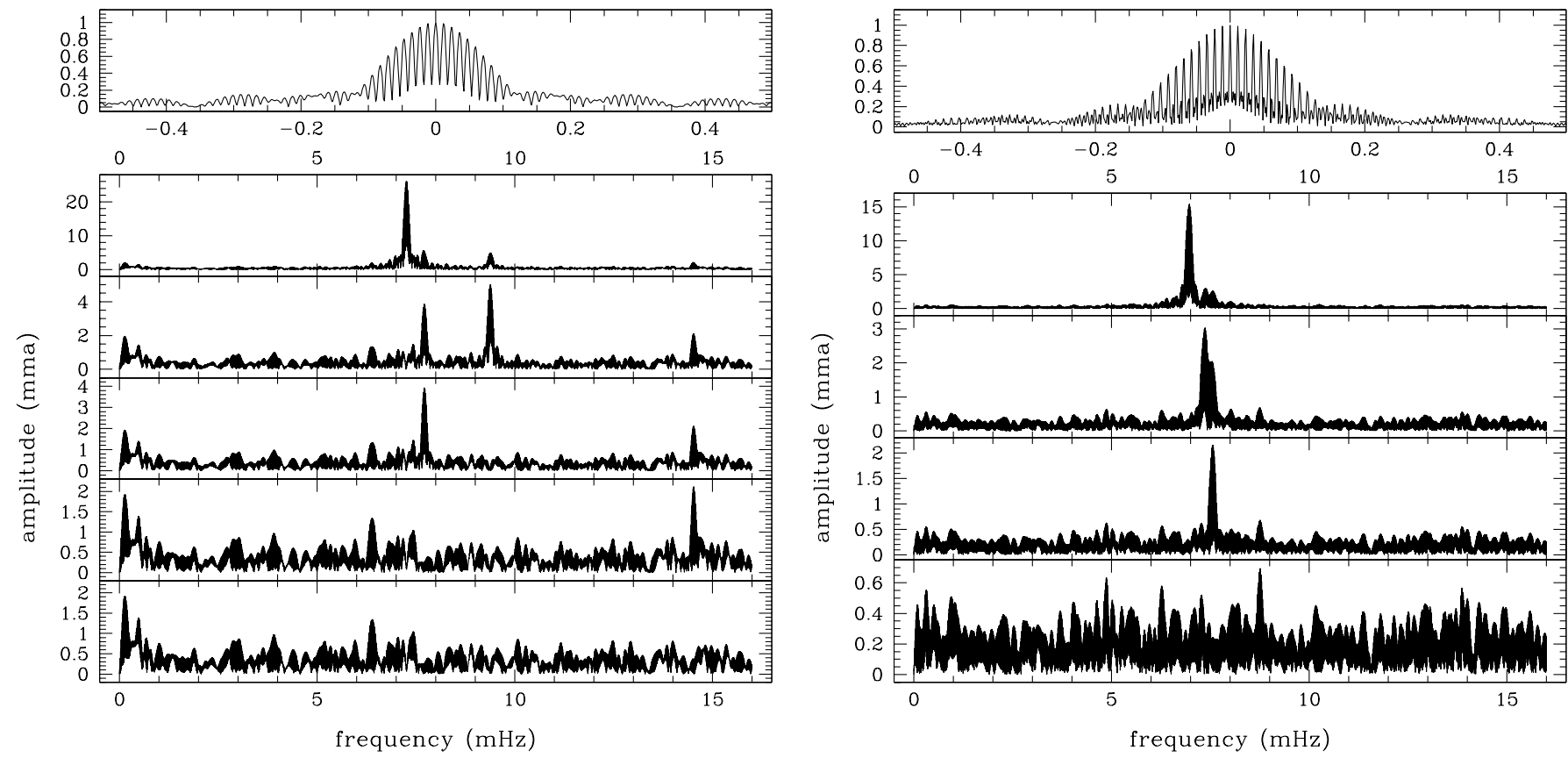

Fig. 4. Prewhitening procedure applied to the combined data sets (all the available nights together). The temporal spectra at each prewhitening step are shown for PG 1325+101 (left) and PG 2303+019 (right). The upper panels represent the spectral windows, i.e. the spectra of a single sinusoid having the same time coverage as the data.

Table 2. Results of the non-linear least-square fits.

\begin{tabular}{|c|c|c|c|c|c|c|c|c|c|c|c|c|c|}
\hline Object & $\begin{array}{l}\text { Date } \\
(2001)\end{array}$ & \multicolumn{3}{|c|}{$(\mathrm{mHz})$} & $F_{4}$ & $P_{1}$ & \multicolumn{2}{|c|}{$(\mathrm{s})$} & $P_{4}$ & $A_{1}$ & $\begin{array}{l}A_{2} \\
(\mathrm{~m})\end{array}$ & $\begin{array}{l}A_{3} \\
\text { a) }\end{array}$ & $A_{4}$ \\
\hline PG $1325+101$ & Jul. 27 & 7.257 & 9.359 & 7.711 & 14.528 & 137.8 & 106.8 & 129.7 & 68.8 & 25.9 & 5.0 & 4.2 & 2.6 \\
\hline & Jul. 28 & 7.258 & 9.384 & 7.711 & 14.522 & 137.8 & 106.6 & 129.7 & 68.9 & 26.0 & 5.2 & 3.7 & 1.8 \\
\hline & Combined & 7.256 & 9.380 & 7.713 & 14.523 & 137.8 & 106.6 & 129.7 & 68.9 & 25.9 & 5.1 & 3.9 & 2.1 \\
\hline \multirow[t]{5}{*}{ PG 2303+019 } & Jul. 26 & 6.963 & 7.357 & 7.531 & & 143.6 & 135.9 & 132.8 & & 16.2 & 4.0 & 3.0 & \\
\hline & Jul. 27 & 6.964 & 7.355 & 7.550 & & 143.6 & 136.0 & 132.5 & & 16.0 & 2.7 & 2.1 & \\
\hline & Jul. 28 & 6.965 & 7.300 & 7.503 & & 143.6 & 137.0 & 133.3 & & 15.7 & 2.3 & 3.3 & \\
\hline & Jul. 29 & 6.961 & 7.344 & 7.548 & & 143.7 & 136.2 & 132.5 & & 15.9 & 3.3 & 2.1 & \\
\hline & Combined & 6.963 & 7.360 & 7.554 & & 143.6 & 135.9 & 132.4 & & 15.9 & 3.2 & 2.2 & \\
\hline
\end{tabular}

In particular PG $1325+101$ is a very interesting object for many reasons: 1) it has large pulsation amplitudes, being third of its class after PG 1605+072 (Koen et al. 1998) and HS 0702+6043 (Dreizler et al. 2001). 2) It shows the shortest pulsation period (68.9 s) among the sdBVs discovered so far. This period is regarded as the first harmonic of the main period at $137.8 \mathrm{~s}$. Up to now first harmonics were almost never seen in sdBV stars; the only case is that one of PG0014+067 (Brassard et al. 2001). 3) Due to its brightness $(B=13.8)$ and its declination, it can be observed even with small telescopes from both hemispheres.

For all these reasons PG $1325+101$ is an ideal candidate for detailed studies and we intend to propose it soon for a large multi-site time-series campaign that may reveal all the high-resolution features of its temporal spectrum.
Acknowledgements. R.S. acknowledges financial support by the MIUR under project "Stellar Pulsation and Evolution" (coordinator V. Castellani). M.A. is supported by the DLR under grant 50 QD 0102, and has been supported by the DFG under grant Bo779/21. The time-series data presented here were obtained using ALFOSC, which is owned by the Instituto de Astrofisica de Andalucia (IAA) and operated at the Nordic Optical Telescope under agreement between IAA and the NBIfAFG of the Astronomical Observatory of Copenhagen.

\section{References}

Brassard, P., Fontaine, G., Billéres, M., et al. 2001, ApJ, submitted

Charpinet, S., Fontaine, G., Brassard, P., \& Dorman, B. 1996, ApJ, 471, L106 
Charpinet, S., Fontaine, G., Brassard, P., et al. 1997, ApJ, 483, L123

Charpinet, S., Fontaine, G., \& Brassard, P. 2001, PASP, 113, 775

Dreizler, S., Schuh, S., Deetjen, J. L., Edelmann, H., \& Heber, U. 2001, A\&A, submitted

Green, R. F., Schmidt, M., \& Liebert, J. 1986, ApJS, 61, 305

Hagen, H-J., Groote, D., Engels, D., \& Reimers, D. 1995, A\&AS, 111, 195

Heber, U., Reid, I. N., \& Werner, K. 2000, A\&A, 363, 198

Kilkenny, D., Koen, C., O'Donoghue, D., \& Stobie, R. S. 1997, MNRAS, 285, 640

Koen, C., O’Donoghue, D., Kilkenny, D., et al. 1998, MNRAS, 296,317

Napiwotzki, R. 1997, A\&A, 322, 256

O'Donoghue, D., Koen, C., Lynas-Gray, A.E., Kilkenny, D., \& Van Wyk, F. 1998, MNRAS, 296, 306
O’Donoghue, D., Koen, C., Kilkenny, D., \& Stobie, R. S. 1999, ASP Conf. Ser., 169, 149

Østensen, R. 2000, Ph.D. Thesis, University of Troms $\varnothing$

Østensen, R., Solheim, J.-E., Heber, U., et al. 2001a, A\&A, 368,175

Østensen, R., Heber, U., Silvotti, R., et al. 2001b, A\&A, 378, 466

Piccioni, A., Bartolini, C., Bernabel, S., et al. 2000, A\&A, 354, L13

Saffer, R. A., Bergeron, P., Koester, D., \& Liebert, J. 1994, ApJ, 432, 351

Saffer, R. A., Livio, M., \& Yungelson, L. R. 1998, ApJ, 502, 394

Silvotti, R., Solheim, J.-E., Gonzalez-Perez, J. M., et al. 2000, A\&A, 359, 1068 